\title{
AC 2008-1503: TEACHING COMPUTATIONAL FLUID DYNAMICS (CFD) TO DESIGN ENGINEERS
}

\section{Junling Hu, University of Bridgeport}

Junling $\mathrm{Hu}$ is an assistant professor in Department of Mechanical Engineering at University of Bridgeport, CT. She teaches courses in the fields of CFD, Thermofluid science, thermal management of electronics, welding engineering, and materials science. Her research area is CFD, transport phenomena in welding processes, and thermal management of electronics. Contact:jjhu@bridgeport.edu

\section{Linfeng Zhang, University of Bridgeport}

Linfeng Zhang is a visiting assistant professor in the Department of Electrical Engineering at the University of Bridgeport, He teaches in the areas of biosensors, controls, signal processing, MEMS, alternative energy. He conducts research in chem/bio sensors design, fabrication, and modeling. Contact: lzhang@bridgeport.edu.

\section{Xingguo Xiong, University of Bridgeport}

Xingguo Xiong is an assistant professor in Department of Electrical and Computer Engineering at University of Bridgeport, CT. He teaches courses in the fields of MEMS (Microelectromechanical Systems), Nanotechnology, VLSI design and testing, semiconductor fabrication, etc. His research interests include VLSI, MEMS and nanotechnology. Contact: xxiong@bridgeport.edu 


\title{
Teaching Computational Fluid Dynamics (CFD) to Design Engineers
}

\begin{abstract}
Computational Fluid Dynamics (CFD) can provide detailed thermal flow information, such as temperature field, pressure field and velocity field, in equipment and process in various industries. Due to the recent rapid growth of powerful computer resources and the development of commercial CFD software packages, CFD has been proven a useful tool for mechanical design engineers.
\end{abstract}

CFD has also gained broad acceptance in the engineering education. It has been adopted in both undergraduate and graduate level courses in many universities. The teaching of CFD in current engineering education can be classified into two types, one is to focus on the numerical methods with little emphasis on using the software and the other is to introduce a CFD software as a virtual reality laboratory in Fluid Mechanics class without emphasis on teaching software. In the first type, students need strong mathematical background to succeed in the class and also need further training to effectively use modern commercial software for real industrial application. While in the second type, students only learned an abstract form of CFD processes, thus they will not be able to use CFD commercial software without further training in this area.

This paper is about the use of CFD in teaching graduate students at this university who were in a two year design track program. Many of these students did not have a good background in mathematics, fluid dynamics, heat transfer, and programming, however, most of them were good at computer aided design in ProE and were very interested in learning CFD as a design tool in industries. STAR-CCM+ was chosen as the CFD software to teach students the entire CFD process in a single integrated software environment. After building a geometry model in ProE, students learned to import the CAD model, set up mesh model, physical model and solver, and postprocess the results in STAR-CCM+. Based on projects, CFD numerical methods and fundamentals of heat transfer and fluid flow were introduced to help students understand the CFD process, interpret, and validate simulation results.

\section{Introduction}

Computational fluid dynamics was introduced in the early 1960s as a specialized engineering tool for the aerospace, defense and nuclear power industries. In the 1970s, CFD spread to the automotive industries and in the ensuing decade grew into a common tool in many commercial applications ${ }^{1}$. Due to the rapid growth of powerful computer resources and the development of general purpose CFD software packages, the last two decades have seen an expanding growth of CFD application in engineering analysis and design. CFD has proven to be a valuable tool to complement experimental findings in flow structure studies ${ }^{2}$. While part of this growth has been within large traditional fluids engineering industries like the aerospace and nuclear industries, a large part of the growth has been in smaller industries or industries that are not necessarily in the fluids engineering mainstream ${ }^{1,2}$. For example, CFD technology is now being used to aid in the design of subway tunnels, cooling systems for densely packed electronic enclosures, helping 
surgeons to understand the fluid flow in human body in hospital, and designing home appliances $^{1,2}$.

Early CFD programs developed before 1980s were almost exclusively for aerospace applications and could be only run on mainframe computers by specialized analysts. These engineers were trained in graduate schools to provide CFD development and application expertise. In 1990s, a host of improved CFD programs with features such as advanced mesh generators, simplified user interfaces, and sophisticated graphics, were developed for new applications in product development and manufacturing ${ }^{3}$. From then, CFD began to be used outside of the research lab by engineers who were not specialists in CFD. Besides the increasing number of industries which adopt CFD in aid of their product design, CFD has played a more important role in the design process. CFD has been used only in the late design stage before final prototyping, but it is a new trend to include CFD within the early design process. Many software developers are now integrating their CFD software with CAD technology to enable design engineers to analyze fluid flow and heat transfer during their design. With minimal training or experience in CFD, design engineer are now required to determine fluid flow, heat transfer, thermal or structural stress, or other physical phenomena in their design process. With the profusion of "easy to use" and "pushbutton" CFD codes for designers, it is easy to get wrong or unphysical CFD predictions without being aware of it $^{4}$. There is a rising issue with the quality and reliability of industrial CFD simulations when more and more companies are integrating their CFD tools into their design processes.

CFD has also gained broad acceptance in the engineering education. It has been adopted in both undergraduate and graduate level courses in many universities. The traditional CFD education has been focused on training specialized analysts with emphasis on algorithms and code development. This was appropriate for training CFD specialist in early days when the commercial codes were less advanced and developed for CFD experts. With the rapid development of commercial packages in the recent twenty years, more CFD analysts are using commercial CFD packages. According to a survey conducted by NAFEMS ${ }^{4}, 80 \%$ of the CFD analysts are using commercial CFD packages, which can simulate many physics and have advanced feature for complex geometry and mesh generation abilities. Students need strong mathematical background to succeed in the traditional CFD class and also need further training to effectively use modern commercial software for real industrial application. Recent developments in CFD curriculum have included commercial packages such as Fluent, Star-CD, CFX as part of the class ${ }^{5-9}$. As the learning curve of commercial packages is steep, many undergraduate curricula ${ }^{10-15}$ introduced teaching CFD software such as FlowLab in Fluid Mechanics class. These teaching software packages have served as a virtual reality laboratory which helped students to understand fluid flow and the internal structure of CFD. However, they cannot prepare students to use commercial CFD software for real application as the teaching software is a much simplified version.

It was challenging to teach CFD to graduate students in a two year design track program at this university. Many of these students did not have a solid background in mathematics, fluid dynamics, heat transfer and computer programming, which are the knowledge required for a traditionally CFD analyst. To effectively teach CFD as a design tool in industries, the class was designed to include lectures, labs, and projects in a commercial code STAR-CCM+. Figure 1 
shows an industrial design process in STAR-CCM+, which is capable of obtaining CFD solution in a single integrated software environment. After building geometry in ProE, students learned the entire CFD process in STAR-CCM+, from importing the CAD geometry, surface wrapping and cleaning-up, generating surface mesh and volume mesh, choosing physical models, monitoring CFD solution, to postprocessing the results. In parallel to the projects in STAR$\mathrm{CCM}+, \mathrm{CFD}$ numerical methods and fundamentals of heat transfer and fluid flow were introduced to help students understand the CFD process and interpret and validate simulation results. This course was not intended to produce CFD specialist, teach only thermal fluids, or use particular commercial software. The purposes of this course were to help students gain understanding of the CFD application in industrial design, the internal structure and operation of CFD solvers, build up their knowledge of fluid mechanics and heat transfer, interpret and validate CFD results, and be aware of the pitfalls of CFD simulations.

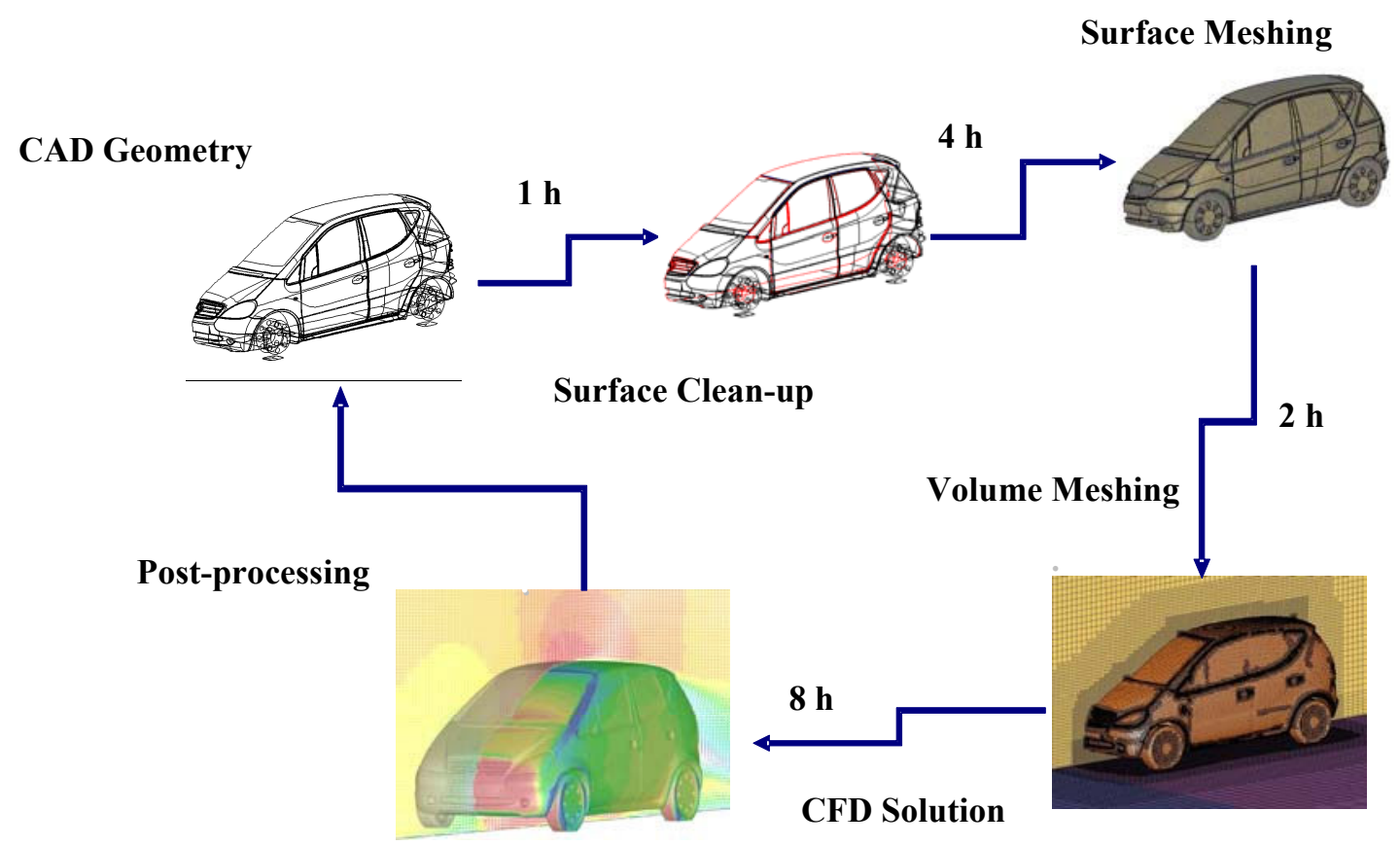

Fig. 1 An application of CFD in industrial design process ${ }^{16}$.

\section{Commercial CFD software STAR-CCM+}

STAR-CCM+ is a new product from CD-adapco, the same company which developed STARCD. STAR-CCM+ is known as the next generation CFD software which was built from scratch with latest software technologies such as client-server architecture, object-oriented programming, scalable parallel performance and supported platforms. It was first released in 2004 and it currently has advanced automated meshing ability, a wide range of physics models, an extensive selection of turbulence models, and powerful post-processing tools. Due to its unique polyhedral cells capability, STAR-CCM+ can be embedded in CAD and PLM environments such as SolidWorks, CATIA V5, Pro/Engineer and NX through its STAR-CAD series products. Compared to other commercial software, STAR-CCM+ is very easy to use in its 
integrated software environment. After geometry generated in CAD is imported into STAR$\mathrm{CCM}+$, mesh generation, physical model and solver set-up, iterative design studies, and post processing are all done in STAR-CCM+.

10 free license seats of STAR-CCM+ were given to the Department of Mechanical Engineering in exchange of user reports, classroom outlines and/or manuscripts in Spring 2007. 35 license seats were purchased for Spring 2008 at academic price. STAR-CCM+ can be easily installed in PCs and licenses can be managed in floating or fixed formats. The user manual, tutorials and help files are well-documented for easy access on each PC. A good set of tutorials is available to guide new users to simulate various industrial fluid flow in a step-by-step format.

\section{Lecture content}

The structure of the lecture was similar to that of Aung ${ }^{7}$. Three textbooks ${ }^{17-19}$, online materials ${ }^{20-}$ ${ }^{21}$, and STAR-CCM+ user manuals ${ }^{16}$ were used as lecture materials in class. The book by John Anderson ${ }^{17}$ was chosen for the first part of the class, including the philosophy of CFD, governing equations, and finite difference method. The book by Patankar ${ }^{18}$ and the book by Versteeg and Malalasekera ${ }^{19}$ were used for finite volume method and solution algorithms. The book by Versteeg and Malalasekera was also used for turbulence modeling and grid generation. Although three books were used by the instructor to develop lecture slides, only the book by Versteeg and Malalasekera was recommended to students. The other two books were reserved in library as references for students. Online lecture slides ${ }^{20,21}$ saved the instructor a lot of time on creating slides, even though many hours were spent on modifying the slides to tailor them to the needs of the class by the instructor. STAR-CCM+ user manual was extensively used in this class, especially on grid generation and turbulence modeling, which currently are the two main issues troubling CFD analysts in the field ${ }^{4}$.

The first week of the course was the introduction of the philosophy of CFD ${ }^{17,20}$. The discussion included what is CFD, why and where it is used, CFD processes, CFD commercial codes, and how CFD works together with analytical fluid dynamics (AFD) and experimental fluid dynamics (EFD) to study fluid mechanics.

Three weeks of the class was devoted to the governing equations of fluid dynamics ${ }^{17}$. The governing equations in partial differential form for the conservation of mass, momentum, and energy were derived in Cartesian coordinates for three dimensional flow. The governing equations were given to students in cylindrical coordinates as well. The physical meaning of each term in the equations was explained and the effects of these terms on the solution procedure of the PDEs were discussed. The governing equations were classified for different types of flows such as viscid, inviscid, Newtonian, Nonnewtonian, compressible, incompressible, transient, and steady flows. The general behavior of the PDEs and their solution methods were discussed based on the mathematical classifications of the governing equations as hyperbolic, parabolic, and elliptic equation. The physical meaning and the importance of boundary and initial conditions in solving each type of the PDE equation were presented.

Five weeks of class were on the discretization of PDEs ${ }^{17-19,21}$, and solution algorithms of discretized equations. Finite difference was introduced first because it could be very easily 
understood by students. A one-dimensional unsteady state diffusion equation was used as an example to illustrate the truncation error, explicit and implicit schemes, and stability associated with discretization. As finite volume method is used in STAR-CCM+ and many other commercial codes, it was introduced in the class despite its difficulty to students. A steady state diffusion equation was used as an example to introduce finite volume method and TDMA was introduced as a solution algorithm for discretized equations. A one-dimensional steady state convection-diffusion problem was used as an example to introduce the different discretization schemes of convection term. Generalized governing equations were then discretized on a staggered grid and SIMPLE algorithm was introduced for the pressure-velocity corrections. Other types of solution algorithms were also introduced to students.

One week of the class was on the grid and mesh generation ${ }^{19}$. The state of art of CFD grid generation was introduced. Structured grid methods, unstructured grid methods, and hybrid grid methods were discussed. STAR-CCM+ user manual on mesh generation was used to help students generate quality mesh. Project was assigned for students to generate different types and sizes of mesh.

Two weeks of the class were on the turbulence modeling ${ }^{19}$. Turbulent flow and turbulence boundary layers were introduced. Turbulence models, including RANS models, LES, DES, and DNS were surveyed. Three RANS models, which are $K-\varepsilon$ model, $\mathrm{V}^{2} \mathrm{f}$ model and RSM model, were studied and compared. STAR-CCM+ user manual on turbulent modeling was referred for their applications, wall treatment, and wall function. The study of the three models was also included in projects.

One week was used to discuss errors and uncertainty in CFD modeling ${ }^{19}$. Roundoff errors, iterative convergence errors, discretization errors, input uncertainty in domain geometry, boundary conditions and fluid properties, and physical model uncertainty were discussed. Verification and validation of CFD simulation and guidelines for best practice in CFD was also introduced.

Other lecture time was used to help students with their projects, including providing the theoretical background related to the projects and interpreting simulation results.

\section{Laboratories and projects}

In the first lab, an introduction demo was shown to students using the Introduction to STAR$\mathrm{CCM}+$ Tutorial. This tutorial does not include mesh generation. A polyhedral mesh file of a symmetric blunt body was imported and used to set up, run, and post-process a transonic flow over a symmetrical blunt body in a wind tunnel. As STAR-CCM+ is a multi-physics continuum based modeling with its unique way to handle physics and mesh, there are some new concepts/terms related to the software. With the help of the user manual, the concepts of continuum, region, boundary, and interface were explained. Students were also directed to the user manual and encouraged to refer to the manual often for understanding, choosing different models, and general problems with operating the software. 
The first project was then assigned to simulate a steady state laminar flow in a pipe and its verification with theoretical results. As meshing models had not been introduced to students, a two-dimensional (2-D) mesh file was provided for running the simulation. In their reports, all students simulated the flow in a pipe as a 2-D flow with wall boundary at both the top and bottom surfaces, as in Fig. 2 (a), which actually simulated a flow between two parallel plates. The problem arose from lack of knowledge in four areas: CFD modeling of space, software operation skills, flow physics, and verification of simulation. Some students were not aware of the difference between the 2-D and axisymmetrical models. While others tried to use the axisymmetric model, but they had troubles with using it in STAR-CCM+. The axisymmetric model in STAR-CCM+ is only for 2-D meshes with restriction on the orientation of the mesh. The restriction requires that the axis should be at $y=0$ in the global coordinate and no part of the mesh can be below $y=0$, as shown in Fig. 2 (b). When students had problem with the axisymmetric model, they used the 2-D model which could give them results. Students were easily satisfied with the parabolic velocity profile for a flow between two plates which was similar to that in a circular pipe. During the validation process, they compared the CFD results with the theoretical values of the maximal $\mathrm{x}$-direction velocity and skin friction coefficient in fully developed region. They found there were $25 \%$ difference between the CFD results and theoretical results. However, they could not evaluate the error due to their limited understanding of the flow physics. From this project, we can see the potential problems in simulation quality and accuracy when more un-prepared CFD users use "easy to use" CFD packages.

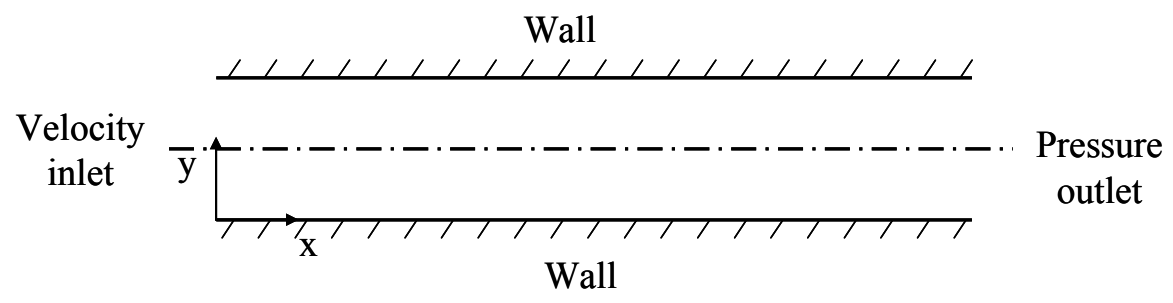

(a)

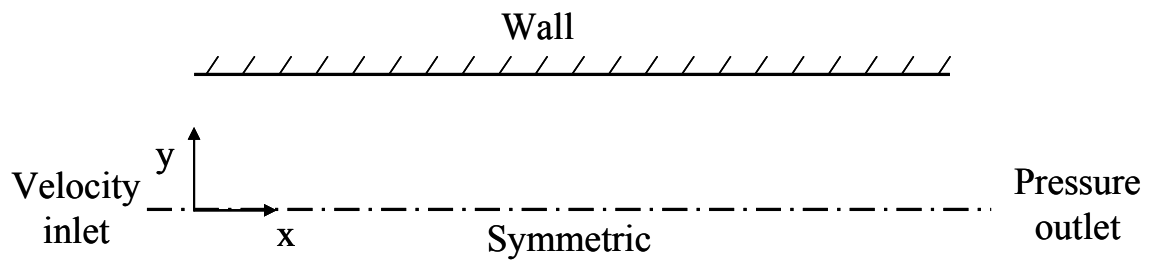

(b)

Fig. 2 Laminar flow between two plates (a) and in a pipe (b).

In the second lab, mesh models were explained and demonstrated using Meshing Tutorial and Meshing manual. Students were now assigned to simulate the steady state laminar flow between two parallel plates and in a pipe with a similar geometry in the first project. The geometry was required to be drawn in ProE and saved in IGS format. The IGS file was then imported into STAR-CCM+ for meshing and simulation. In the project, student needed to generate a quarter of a 3-D cylinder in ProE, imported it as a surface in STAR-CCM+, translated and rotated the geometry to the appropriate orientation to generate mesh for the axisymmetric model. A 3-D 
volume mesh was first generated after selecting mesh models and defining reference properties and boundary meshing properties. The 3-D volume mesh was then converted to 2-D mesh for the axisymmetric model, as shown in Fig. 3. From the report of the first project, we found students need detailed guidance with analyzing results and writing report. Before the project, the theoretical background in channel flow was explained and so were its important features, such as maximal velocity in $\mathrm{x}$ direction, skin friction coefficient, developing length, velocity profile, etc. With better understanding of the models and better capability to expect what will to be studied, students successfully finished the project and had a better understanding of the flow physics and the verification of the CFD results.

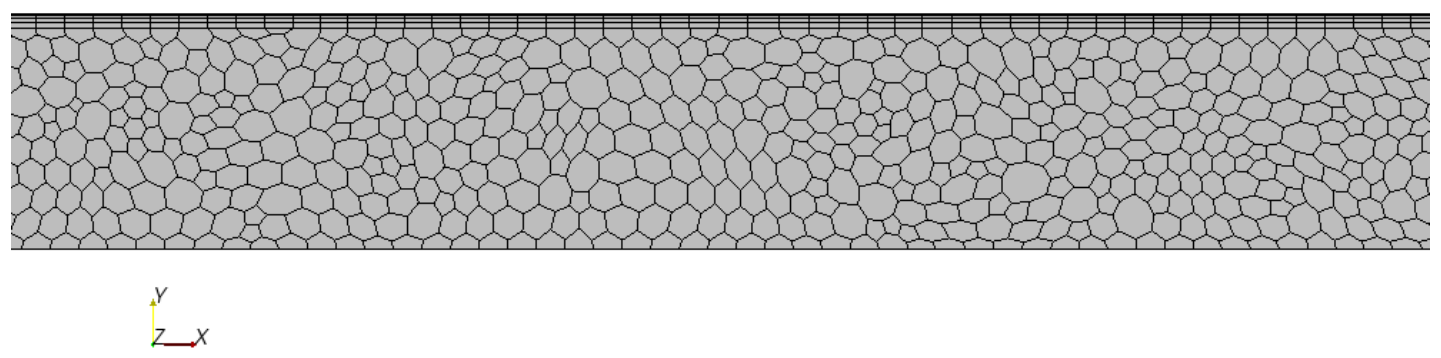

Fig. 3 A 2-D polyhedral mesh for modeling flow in a pipe.

The other four projects were taken from the class website of Stern ${ }^{20}$ at University of IOWA. Their detailed lab materials including lab instructions, lab report instructions, and experimental data were adopted in class. These four projects were well developed laboratory exercises designed to teach student CFD methodology with educational interface in FlowLab. Even though they were designed for FlowLab user in a much simplified interface, students benefited from the templates with better understanding of CFD process and flow physics. The lab materials served as a very good guidance for students in their labs and writing reports. These materials saved instructor time developing tutorials to enhance understanding of flow physics. As these lab instructions were prepared for FlowLab, extra efforts were needed from students in preprocessing, model selections, and postprocessing. These projects were also modified to fit with other part of the class, such as different turbulent models and mesh generation.

The following are the names and project descriptions slightly modified from the four laboratory exercises taken from the website of $\operatorname{Stern}^{20}$ :

Project 1 Verification of laminar and validation of turbulent pipe flows Students will have "hands-on" experiences using STAR-CCM+ to compute axial velocity profile, centerline velocity, centerline pressure, and skin friction coefficient. Students will conduct verification studies for skin friction coefficient and axial velocity profile of laminar pipe flows, including iterative error and grid uncertainties, and effect of refinement ratio on verification. Students will use post-processing tools (streamlines, velocity vectors, contours, $x-y$ plots, streamlines, and tables) to visualize and analyze the flow fields. Students will validate turbulent pipe flow simulation using EFD data and analyze the differences between laminar and turbulent flows.

Project 2 Verification and validation of turbulent flow around a clark-y airfoil 
Students will have "hands-on" experiences using STAR-CCM+ to conduct verification and validation for lift coefficient and pressure coefficient distributions, including effect of numerical scheme. Students will manually generate the "O" type and " $\mathrm{C}$ " type meshes and investigate the effect of domain size and effect of angle of attack on simulation results. Comparisons between inviscid and viscous flows will also be conducted. Students will use post-processing tools (streamlines, velocity vectors, contours, $\mathrm{x}-\mathrm{y}$ plots, streamlines, and tables) to visualize and analyze the flow fields. Students will analyze the differences between CFD and EFD and analyze possible source of errors.

Project 3 Simulation of turbulent flow in an asymmetric diffuser Students will have "hands-on" experiences using STAR-CCM+ to conduct verification and validation for total pressure change, velocity and turbulent kinetic energy, skin friction coefficient, etc. Effect of turbulent models will be investigated, with/without separations. Students will manually generate meshes, solve the problem and use post-processing tools (contours, velocity vectors, $x-y$ plots, streamlines, tables, and user defined filed functions) to visualize and analyze the flow field. Students will analyze the differences between CFD and EFD.

Project 4 Simulation of turbulent flow over the ahmed body Students will have "hands-on" experiences using STAR-CCM+ to investigate the effect of slant angles (25 and 0 degrees), effect of meshes (coarse and fine) on simulation results by comparing with experimental data. Students will use post-processing tools (streamlines, velocity vectors, contours, $\mathrm{x}-\mathrm{y}$ plots, streamlines, animations, and tables) to visualize and analyze the mean and instantaneous flow fields and compute the shedding frequency (Strouhal number). Students will analyze the differences between CFD and EFD.

These four projects were assigned as term project to students. Each project was divided and assigned to two or three groups with each group working on one part. The submission of the project was also divided into five different phases, including deadlines for project proposal, geometry generation, mesh generation, simulation results, and final report. The problems revealed in the reports were discussed in the class. The final project was presented by each group, so students not only had hands on experience on the part they were working on, but also could learn a more complete picture of CFD techniques from the works of others.

\section{Discussions}

We observed that students could quickly obtain enough knowledge of STAR-CCM+ to investigate fluid flow with the guidance of the instructor. They could generate geometry in ProE, import the geometry as a surface, generate appropriate meshes, set up physical models, solve numerical problems, visualize and analyze the flow field with post-processing tools. However, it was still very difficult for students to model a new flow phenomenon alone. This was what we have expected at the beginning as we were not intended to produce experts. CFD experts should still be the ones to develop CFD models for new industrial applications, while design engineers will perform the simulation and interpret the results with guidance. 
Students preferred STAR-CCM+ as a teaching tool over FlowLab used in my fluid dynamics class because STAR-CCM+ is a commercial package used in industry. They were amazed by the ability of STAR-CCM+ in modeling different geometry, generating mesh, choosing physical models and postprocessing the results. They were glad that they gained experience in operating the software, especially in postprocessing results, but on the other side, they were overwhelmed by the complexity of choosing physical models.

The lectures were prepared to help students understand the fundamental numerical techniques and models. From the tests on the lectures, we found students had more problems with the lectures than with the projects. The lectures were much simplified compared to those for the traditional CFD courses, but they were still similar in including all of the important fundamental numerical techniques. As the traditional CFD courses were designed for CFD developers, the similar lectures in this class were still difficult for beginners, especially those who did not have a good background in mathematics, fluid dynamics, and heat transfer. We will continue to modify the lectures to make them easier to understand while at the same help students grasp the essential CFD internal structures.

A formal survey was not conducted for this class. However, discussions were often held with students on the effectiveness of the lecture contents and projects. From the feedbacks, we found the method of combining user manual with lectures and projects help students to understand the fundamentals. From the feedbacks, we found we had reached the purposes of this course, which were to help students gain understanding of CFD application in industry design, the internal structure and operation of CFD solvers, build up their knowledge of fluid mechanics and heat transfer, interpret and validate CFD results, and be aware of the pitfalls of CFD simulations.

The instructor found this course was very time consuming in developing new lecture materials on grid generation and turbulence modeling, projects preparation, and helping students with projects. STAR-CCM+ was introduced very late - after 4 weeks of class. It should have been introduced at the beginning of class to give students more time to know the software and understand the modeling in the software with the lectures. It was also because the time limit that another project on heat transfer was not able to be covered in the class. We expect the teaching of this course in Spring 2008 would be more effective with some academic teaching modules ${ }^{16}$ developed for STAR-CCM+ by the software developer.

\section{Conclusions}

This paper presents the design and implementation of Computational Fluid Dynamic (CFD) course in the Department of Mechanical Engineering at this university for graduate students in a two year design track program. With the combination of lecture, labs, and projects, we have introduced CFD as a design tool to students who did not have a good background in mathematics, fluid dynamics, heat transfer, and programming. We had also reached the purposes of this course, which were to help students gain understanding of CFD application in industry design, the internal structure and operation of CFD solvers, build up their knowledge of fluid mechanics and heat transfer, interpret and validate CFD results, and be aware of the pitfalls of CFD simulations. 


\section{Bibliography}

1 J. Thilmany, "How Does Your Fluid Flow", Mechanical Engineering Magazine, 2003.

${ }^{2}$ G.D. Stubley, "CFD for Undergraduate Engineering Students", http://www.cfdsc.ca/bulletins/07/index.html, 1996.

3 A. Wolfe, "CFD software: pushing analysis to the limit", Mechanical Engineering-CIME, January, 1991.

${ }^{4}$ Tim Morris, "Quality and Reliability in Engineering CFD Simulations", Fluent News, 2005.

${ }^{5}$ H.K Navaz, B.S. Henderson, and G. Mukkilmarudhur, etc., "Bring Research and New technology into the Undergraduate Curriculum: A Course in Computational Fluid Dynamics", Proc. 1998 ASEE Annual Conference proceedings, June, Seattle, Washington, 1998.

${ }^{6}$ Guessous, L., Bozinoski, R., Kouba, R. and Woodward, D., "Combining Experiments with Numerical Simulations in the Teaching of Computational Fluid Dynamics," Proc. 2003 ASEE Annual Conference \& Exposition, June, Nashville, Tennessee, 2003.

${ }^{7}$ K. Aung, "Design and Implementation of an Undergraduate Computational Fluid Dynamics (CFD) Course," Proc. 2003 ASEE Annual Conference \& Exposition, June, Nashville, Tennessee, 2003.

${ }^{8}$ D. Pines, "Using Computational Fluid Dynamics to Excite Undergraduate Students about Fluid Mechanics", Proc. 2004 ASEE Annual Conference \& Exposition, June, Lake City, Utah, 2004.

${ }^{9}$ R. Bhaskaran, L. Collins, "Integration of Simulation into the Undergraduate Fluid Mechanics Curriculum using FLUENT", Proc. 2003 ASEE Annual Conference \& Exposition, June, Nashville, Tennessee, 2003.

${ }^{10}$ R. D. LaRoche, B. J. Hutchings, R. Muralikrishnan, "FlowLab: Computational Fluid Dynamics (CFD) Framework for Undergraduate Education”, Proc. 2002 ASEE Annual Conference \& Exposition, June, Montreal, Quebec, CA, 2002.

${ }^{11}$ Appanaboyina, S. and Aung, K., , "Development of a VRML Application for Teaching Fluid Mechanics," Proc. 2004 ASEE Annual Conference \& Exposition, June, Salt Lake City, Utah, 2004.

${ }^{12}$ R. Jia, S. Xu, S. Gao, EL-S. Aziz, S. Esche, and C. Chassapis, “A Virtual Laboratory on Fluid Mechanics,” Proc. 2006 ASEE Annual Conference \& Exposition, June, Chicago, Illinois, 2006.

${ }^{13}$ R. LaRoche, B. Hutchings, and R. Muralikrishnan, "FlowLab: Computational Fluid Dynamics (CFD) Framework for Undergraduate Education," Proc. 2002 ASEE/SEFI/TUB Colloquium, 2002.

${ }^{14}$ F. Stern, T. Xing, D. Yarbrough, A. Rothmayer, G. Rajagopalan, S.P. Otta, D. Caughey, R. Bhaskaran, S. Smith, B. Hutchings and S. Moeykens, "Development of Hands-On CFD Educational Interface for Undergraduate Engineering Courses and Laboratories," Proc. 2004 ASEE Annual Conference \& Exposition, June, Salt Lake City, Utah, 2004.

${ }^{15}$ F. Stern, T. Xing, M. Muste, D. Yarbrough, A. Rothmayer, G. Rajagopalan, D. Caughey, R. Bhaskaran, S. Smith, B. Hutchings and S. Moeykens, "Integration of Simulation Technology into Undergraduate Engineering Courses and Laboratories", Int. J. Learning Technology, Vol. 2, No. 1, pp.28-48, 2006.

${ }^{16}$ STAR-CCM+ user manual and Academic Course help files.

${ }^{17}$ J.D., Anderson, "Computational Fluid Dynamics: The Basics with Applications," McGraw Hill, 1995.

${ }^{18}$ S.V. Patankar, "Numerical Heat Transfer and Fluid Flow," McGraw Hill, 1980.

${ }^{19}$ H.K. Versteeg, and W. Malalasekera, "An Introduction to Computational Fluid Dynamics: The Finite Volume Method," Prentice Hall, 1995.

${ }^{20} \mathrm{http}: / /$ css.engineering.uiowa.edu/ me $160 /$

${ }^{21} \mathrm{http}: / /$ azkef.org/ChE490/ 